\title{
Genome wide association identified colorectal cancer susceptibility loci and colorectal cancer risk in Lynch syndrome
}

\author{
Mala Pande*, Marsha L Frazier, Patrick M Lynch, Russell Broaddus, Christopher I Amos \\ From 13th Annual Meeting of the Collaborative Group of the Americas on Inherited Colorectal Cancer \\ Honolulu, Hawaii, USA. 16-17 October 2009
}

\section{Background}

Recent genome-wide association studies (GWAS) have identified several common low-risk variants for colorectal cancer. Although some of the GWAS were enriched for young onset and family history positive colorectal cancer cases, it is not clear if these variants modify colorectal cancer risk for people with Lynch syndrome. In a case-unaffected sibling analysis of population and clinic based sibships, a study by Poynter et al. (2007) found a stronger association for risk variants in the 8q24 region (rs10505477 and rs6983627) with microsatellite instability (MSI)-high tumors. MSI-high tumors are characteristic of Lynch syndrome. Therefore we hypothesize that these variants may also influence colorectal cancer risk in Lynch syndrome. Recently Wijnen et al. (2009) have reported on some of the GWAS risk variants in Dutch Lynch syndrome families and although they did not find an association between rs6983627 and colorectal cancer risk, other risk variants in 8q24 and 9p24 have yet to be characterized in Lynch syndrome.

\section{Methods}

In a retrospective cohort study design we analyzed 267 Lynch syndrome subjects from the M. D. Anderson Lynch syndrome registry with a proven DNA mismatch repair gene mutation. We genotyped 3 risk variants: 8q24 (rs10505477: $\mathrm{T}>\mathrm{C}$ and rs6983627: $\mathrm{T}>\mathrm{G}$ ) and 9p24 (rs719725: A $>$ C) and used Cox proportional hazards regression (Hazard ratios and 95\% confidence intervals) to analyze the association between each of the risk variants and colorectal cancer risk while adjusting for sex and familial correlation.

\footnotetext{
* Correspondence: mpande@mdanderson.org
The University of Texas M. D. Anderson Cancer Center, Houston Texas 77030,

* Correspondence: mpande@mdanderson.org
The University of Texas M. D. Anderson Cancer Center, Houston Texas 77030, USA
}

\section{Results}

Of the 267 subjects from 120 families with 11767 person years of follow-up, 138 had colorectal cancer and the remaining was unaffected. We modeled each of the 3 variants as codominant, additive and recessive but none of them were associated with colorectal cancer risk in our cohort.

\section{Conclusion}

Although the GWAS identified colorectal cancer risk variants are potential modifiers of colorectal cancer risk in Lynch syndrome, it is possible that the lack of association seen in our data is due to our study being underpowered to detect the modest association typically associated with these common variants. Further studies with a larger sample size are warranted to confirm presence or true lack of an association.

\section{Acknowledgements}

This research was supported in part, by a cancer prevention fellowship supported by the National Cancer Institute grant R25T CA57730, Robert M. Chamberlain, Ph.D., Principal Investigator. Research also supported by CA 070759, Marsha L. Frazier, Ph.D., Principal Investigator

Published: 25 May 2010

doi:10.1186/1897-4287-8-S1-P16

Cite this article as: Pande et al:: Genome wide association identified colorectal cancer susceptibility loci and colorectal cancer risk in Lynch syndrome. Hereditary Cancer in Clinical Practice 2010 8(Suppl 1):P16. 